\title{
Handedness and the Preference of the Visual Field in Face Perception
}

\author{
Slávka Démuthová, (assoc. prof., Ph.D.) \\ University of Ss. Cyril and Methodius in Trnava, Slovakia \\ Andrej Démuth, (prof., Ph.D.) \\ Trnava University in Trnava, Slovakia
}

Doi: 10.19044/esj.2018.c3p8 URL:http://dx.doi.org/10.19044/esj.2018.c3p8

\begin{abstract}
Face perception is tightly connected with the hemispherical dominance, and thus can be different in right-, left-handed and ambidextrous subjects. The study focuses on preferences for the left or the right visual field in face perception by testing the preference for the left or the right half of the chimeric face, which has been blended from half male and half female facial composite. The preference in evaluation process of the chimeric face has been studied on 2,207 participants $(59.9 \%$ female; 6,8\% left-handers and 6,5\% ambidextrous; mean age $=24.08$ years) with an emphasize on their handedness and sex. Chi-Square Test showed a significant preference (Asymp. sig = 0.000) for the left visual field in right-handed, but not in left-handed (Asymp. sig. $=0.072)$ and ambidextrous (Asymp. sig $=0.134)$ participants. The same results were gained also after evaluation for each sex separately with an exception for ambidextrous females. They preferred left visual field in face evaluation statistically significantly (Asymp. sig. $=0.022$ ) more than the right. The suggestions for further research and limitations of the study are discussed.
\end{abstract}

Keywords: Face perception, visual field, handedness, hemisphere dominance, chimeric face

\section{Introduction}

Psychologist and biologists within the research of human body's functioning constantly reveal, that bilateral pairs of structures in the body are not symmetrical in either form or function (Porac and Coren 1976). Usually, one of the structures in the pair is behaviourally or physiologically superior to the other, with the superior side being referred to as dominant (Jung et al. 2017). In visual perception, the dominance of the left visual field is known as the left-visual field (LVF) superiority (Le Grand, Mondloch, Maurer, and Brent 2003; Thomas et al. 2008; Yovel, Tambini, and Brandman 2008). This 
tendency is very pregnant also in face perception - it is proved, that the left half of perceived face (from the position of the observer) is dominant and more important as the right half for the majority of observers in various task (e.g., in the evaluation of identity, gender or age - Burt and Perrett 1997; Bourne and Gray 2011; Dole, Méary, and Pascalis 2017).

It is suggested, that the LFV superiority is based on biological mechanisms that are tight to different lateralization of functions in the brain. For example, processing of words is strongly lateralized in the left brain hemisphere (Cohen et al. 2000; Dehaene and Cohen 2011), whereas face perception is dominantly tight with the right hemisphere (Burt and Perrett 1997; Yovel, Tambini, and Brandman 2008). The connection of the right hemisphere $(\mathrm{RH})$ with the LVF lies in the process of visual perception information from the left visual field is sent to the right hemisphere, whereas information from the right visual field is sent initially to the left hemisphere (Jung et al. 2017).

Researches investigating the effect of the lateralization of brain functions on face perception revealed, that e.g. information about the face retrieved from the LVF better predicted attractiveness ratings of faces than from the right visual field (Franklin and Adams 2010). Also, it has been detected, that the left half of the face seems to be crucial also for emotions detection better than the right half (Nicholls, Wolfgang, Clode, and Lindell 2002; Chen, Liu, and Fu 2007). However, results are not always consistent with the presumption of the general preference of the left part of the face within face perception - e.g. Zaidel and Cohen (2005) did not reveal any significant differences in the preference for the left or the right half of the face in attractiveness assessment of left-left and right-right mirror facial composites. In another study, Zaidel with colleagues found, that subjects significantly rated the right-right composite of ordinary women's faces as being more attractive than the left-left, whereas men's right-right versus leftleft facial mirror composites evaluation was not significantly different (Zaidel, Chen, and German 1995).

\section{Problem}

Studies dealing with the face perception and the LVF dominance enriched the area of interest for variables that may intervene with the preference of the LVF and could explain the controversial results. They focused on the sex of the evaluator, on the sex of the evaluated face (Zaidel, Chen, and German 1995), eye dominance (Jung et al. 2017), or on the various types of evaluation tasks (Chen, Liu, and Fu 2007; Franklin and Adams 2010). From the result it seems, that investigation in similar areas is needed. There are suggestions to evaluate the variable of handedness (Jung et al. 2017), which has often been omitted from researches. Clear right-hand preference is 
the manifestation of the typical brain functional lateralization and applies to the majority of population (approximately 90\% - Denny and Zhang 2017). Therefore, the left-handedness may point to brain lateralization variations that may possibly cause also the variation in functions connected with the face perception. Similarly, ambidexterity, in the sense of being equally good at a particular task with both hands, can be associated with different preferences for left or right visual field in the face perception. Some researchers even suggest (see e.g. Crow, Crow, Done, and Leask 1998) that this equal skill is a marker for failure to develop cerebral dominance of either hemisphere and may be the cause of various cognitive deficits.

Therefore, we decided to test, whether the left-handers show different preferences for the visual field when evaluating the human faces than the righthanders or the ambidextrous and whether revealed tendencies apply equally for male and female evaluators.

\section{Procedure and Methods}

The participants took part in the research voluntarily. After a short exposition of the main ideas of the research and after granting oral consent they continued by completing a battery of questionnaires, tests and sets of questions and tasks. Only the main area of research was disclosed to the participants, otherwise they were blind to the aims of the specific tasks and questions.

Preference for the left/right half visual field has been detected by two facial composites (Fig. 1). The subjects were asked to judge, which of the faces in Fig. 1 was the more feminine. In reality, each face is half woman and half man. In this chimeric face, the halves are subtly blended across the midline so that the observer does not notice the join. The first (top) face composite is blended from the left male and right female half of the face, whereas the second (bottom) face composite is made from the left female and right male half of the faces. The two facial composites are therefore the same except that they are mirror reflections (Perrett 2010). This task reveals which half of the face the subject preferred for when making decisions on human faces and consequently points to the visual fiela that is dominant for the face perception.
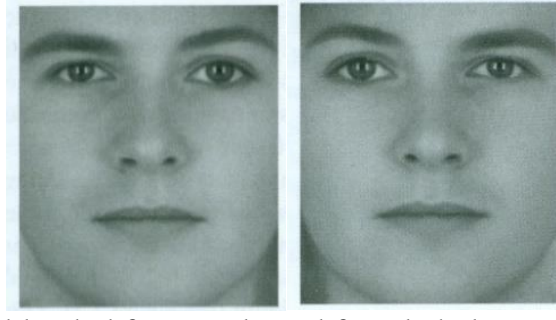

Fig. 3 Facial composites blended from male and female halves of the face (Perrett 2010, p. 29) 
Data on age, sex, and handedness were entered into the test battery by participants. Age was stated in years. Participants had to choose between the options: "male"/"female". Within the handedness detection, the participants finished the sentence: "When performing activities as writing, cutting, throwing... etc. you dominantly use:" by choosing from three options: "right hand", "left hand", "both hands".

\section{Subjects}

Subjects enrolled in the research on a voluntary basis. Out of 2,408 participants, $201(8,35 \%)$ were excluded from further evaluation due to incomplete or incorrectly completed questionnaires. The final sample $(\mathrm{N}=$ $2,207)$ consisted of Slovak females $(\mathrm{N}=1,323 ; 59.9 \%)$ and males. The majority of the participants were the right-handers $(\mathrm{N}=1,913 ; 86,7 \%) ; 6,8 \%$ were left-handers $(\mathrm{N}=150)$ and $6,5 \%(\mathrm{~N}=144)$ were ambidextrous. The mean age of the sample was 24.08 years with a minimum of 17 and a maximum of 72 .

\section{Results}

A general tendency to assess the human face using the left visual field was present also in our sample - a statistically significant (Asymp. sig. $=$ 0.000 ; chi-sq. $=72.135)$ majority $(\mathrm{N}=1303)$ relied on the left half of the face in face evaluation. However, when dividing the sample into three groups of right-, left-handed and ambidextrous subjects, the results vary (Table 1).

\begin{tabular}{|c|c|c|c|c|c|}
\hline \multicolumn{6}{|c|}{ Right-handed participants } \\
\hline Preference for: & $\begin{array}{c}\text { Observed } \\
\mathrm{N}\end{array}$ & Expected N & Residual & Chi-sq. & $\begin{array}{c}\text { Asymp. } \\
\text { sig. }\end{array}$ \\
\hline Right half of the face & 777 & 956.5 & -179.5 & \multirow{2}{*}{67.371} & \multirow{2}{*}{0.000} \\
\hline Left half of the face & 1136 & 956.5 & 179.5 & & \\
\hline Total & 1913 & & & & \\
\hline \multicolumn{6}{|c|}{ Left-handed participants } \\
\hline Right half of the face & 64 & 75.0 & -11.0 & \multirow{2}{*}{3.227} & \multirow{2}{*}{0.072} \\
\hline Left half of the face & 86 & 75.0 & 11.0 & & \\
\hline Total & 150 & & & & \\
\hline \multicolumn{6}{|c|}{ Ambidextrous participants } \\
\hline Right half of the face & 63 & 72.0 & -9.0 & \multirow{2}{*}{2.250} & \multirow{2}{*}{0.134} \\
\hline Left half of the face & 81 & 72.0 & 9.0 & & \\
\hline Total & 144 & & & & \\
\hline
\end{tabular}

Tab. 1. Chi-Square Test for the distribution of choices for the left or the right half of the face in face evaluation

The right-handers showed usual preference for the face evaluation depending on the left half of the face (LVF superiority) and this preference was statistically significant (Asymp. sig. $=0.000$ ). There is also a tendency to prefer the left half of the face in the groups of left-handers and ambidextrous 
participants, however it is not statistically significant (Asymp. sig. for lefthanders $=0.072$ and Asymp. sig. for ambidextrous subjects $=0.134$ ) and cannot be expected generally with such high probability as in the right-handed participants.

In the next step, we examined the preference for the visual field in the groups of right-and left-handers, and ambidextrous participants separately for male and female sex. Both, male and female right-handed participants showed a clear, statistically significant (Asymp. sig. in both cases $=0.000$ ) preference for the left half of the face and thus LVF superiority. Results from the ChiSquare Test in left-handed males and females show, that the slight tendency to prefer left half of the face in face evaluation is not statistically significant (in male sample Asymp. sig $=0.085$; Chi.-sq. $=2.965$; in female participants Asymp. sig. =0.351; Chi.-sq. =0.871). Difference between sexes is present in ambidextrous participants - whereas the preference for the left half of the face was not statistically significant in male evaluators (Asymp. sig. $=0.808$; Chi.sq. $=0.059)$, female evaluators preferred left half of the face statistically significantly (Asymp. sig. $=0.022$; chi-sq. $=5.263$ ).

\section{Discussion}

Results from our research support previous findings reporting the preference of left half of the chimeric face within the face perception (Perrett 2010; Bourne and Gray 2011; Dole, Méary, and Pascalis 2017). These findings are consistent with the basic information on functional lateralization of the brain. Face perception belongs to the functions that are hemispherically lateralized (Ferneyhough, Stanley, Phelps, and Carrasco 2010) with the dominance of the right hemisphere (Luh, Redl, and Levy, 1994; Thomas et al. 2008; Yovel, Tambini, and Brandman 2008). However, this hemisphere dominance and the lateralization of brain function is not universal; it applies mainly to right-handers. Left-handers show more intersubject variability in these lateralized brain functions (e.g., Dronkers and Knight 1989; Luh, Redl, and Levy 1994). Some authors even suggest, that the hemispheres of ambidextrous and left-handed people's brains are almost symmetric and that the equal use of hands in ambidextrous subjects is a marker for failure to develop cerebral dominance of either hemisphere (e.g., Crow, Crow, Done, and Leask 1998). It seems, that the results of our study are in concordance with these ideas - whereas the right-handers showed a statistically significant preference for the left half of the chimeric face (and thus for the LVF), lefthanders and ambidextrous participants did not. Presumptions about the importance of the effect of handedness on visual field preference within the face perception (Jung et al. 2017) seems to be legitimate. We therefore suggest the further research in the area of handedness and face perception. 
When we focused on the sex of the evaluators, the results did not differ, except for one case. In ambidextrous participants the significant preference for the left half of the face has been present in female participant. According to a very low number of ambidextrous women $(\mathrm{N}=76)$ compared to the righthanded female $(\mathrm{N}=1,154)$ in our sample, the interpretation of this finding should be very cautious. Some authors (e.g., Denny and Zhang 2016) suggest, that the dominance of the hemisphere (and consequent hand dominance) is not a nominal variable, but is rather cardinal. From this point of view, the handedness is a continuum ranging from right handedness to left handedness. Within this perspective, ambidexterity is a middle step, therefore the fact that it showed a greater preference for the left half of the face than in left-handed females seems logical. When we rank the values of significance of the preferences for the left side of the face in female participants gained in the Chi-Square Test, the order is left-handed (Asymp. sig. =0.351), ambidextrous (Asymp. Sig. = 0.022), and right-handed (Asymp. Sig. = 0.000), which corresponds with the continuum left-handedness - ambidexterity - righthandedness. However, this fact does not explain the finding, why the preference for left half of the face in ambidextrous participants was present on significant level in female, but not male participants. We can further assume, that except the different functional lateralization of the brain according to the dominance of the hemisphere, it is known, that also the sex of the subject plays an important role. It is possible, that a specific combination of sex and brain dominance resulted in such a preference. However, further evaluation of this suggestion is definitely needed. Also, within such a small sample of ambidextrous women, already mentioned intersubject variability of functional lateralization in ambidextrous subjects (Dronkers and Knight 1989; Luh, Redl, and Levy 1994) could play the crucial role.

However, there are more possible explanations, too that point to the limits of our study. We did not detect the situational context under which ran the evaluation process of chimeric faces. The participants were not asked whether they evaluated femininity of presented faces in neutral context, or whether they considered this task as connected with attractiveness task or as the evaluation of potential sexual partner. As Franklin and Adams (2010) state, face evaluation in a nonsexual context is depended more on the right than on the left half of the face. Also, the prevalence of ambidextrous participants was atypical in our sample. Similar studies executed at the same time recorded proportions of right, left or mixed handed subjects $82.8 \%, 10.3 \%$ and $6.9 \%$ (Denny and Zhang 2017). In our sample, the portion of left-handed and ambidextrous participants was nearly the same (6,8\% and 6,5\%). One possible explanation lies in the fact, that even though in Slovakia there are not anymore trends to convert left-handers to right-handers, there are still some conditions present that favour right-handed subjects. This fact could lower the number of 
left-handers. Also, we did not ask subjects, whether they did not convert from left-handedness to right-handedness in childhood. Specialized researches show, that even when there could be no differences in the manual performance of "natural" vs. "converted" right-handers, the differences in brain activity still persist even in adulthood (Siebner et al. 2002). This finding correspondents with the fact, that many aspects of face perception are biologically set and have an inborn character (Maguinness and Newell 2014). Also several other studies point to the fact, that face processing is more closely related to innate factors (Reiss and Reiss 1997). More recent (Grabowska et al. 2012) magnetic resonance studies show, that switched individuals share features of both lefthanders and right-handers regarding their motor control architectures of brain.

Another limit of the study is, that the hand dominance has not been tested; data were gained by the self-evaluation of subjects. It is possible, that self-detected handedness does not correspond with the real facts. Therefore, we suggest in future research to test the handedness by one on available tests (e.g., Sheard 1957; Oldfield 1971 or its updated version by Cohen 2008). Within the subject of consequences of hemispheric dominance, an evaluation could be also targeted to eye dominance as this variable has been proved do correlate with the preference of the visual field (Jung et al. 2017). From the previous studies it is also obvious, that a mental health of participants may play an important role, too. E.g., individuals with autism are known by deficits in attending to faces and in their face-processing abilities. Detailed research showed, that they begin to explore the face by looking at the eye in the visual field ipsilateral to their dominant eye, whereas healthy adults begin to explore the face by looking at the eye in the visual field contralateral to their dominant eye (Hernandez et al. 2009). Also, patients with schizophrenia prefer to view the right side of the face first (Phillips and David 1997). It therefore seems, that research focused on the preference of perception of faces has to be complex and should take into account many variables.

\section{Conclusion}

Within the brain functioning, the processes of the face perception are mostly lateralized and dominantly connected with the right hemisphere. However, the hemispheric dominance that is manifesting externally by e.g. the contralateral handedness or by the dominance of bilateral pairs of structures in the body is not the same for all subjects. Therefore, the investigation of the face perception, of the preferences and specificities of such examination should include also variables as handedness, or eye dominance. Our research on the sample of 2,207 participants showed a clear, statistically significant left visual field superiority in face perception in right-handed participants. This strong preference was not proved in left-handed and ambidextrous subjects 
except the group of ambidextrous women. We suggest future investigation within the area of preference of left visual field in face perception focused on other variables.

\section{Acknowledgements}

This work was supported by the Slovak Research and Development Agency under the contract No. APVV-15-0294 and by the grant KEGA 004TTU-4/2015.

\section{References:}

1. Bourne, V. J. and D. L Gray. One face or two? Contrasting different versions of the chimeric faces test. Laterality 16 (5), 2011, pp. 559564, doi: 10.1080/ 1357650X.2010.498119.

2. Burt, D. M. and D. I. Perrett. Perceptual asymmetries in judgements of facial attractiveness, age, gender, speech and expression. Neuropsychologia, 35 (5), 1997, pp. 685-693.

3. Cohen, M. S. Handedness Questionnaire. 2008 [Online]. Retrieved from: URL <http://www.brainmapping.org/shared/Edinburgh.php> [2017-28-08].

4. Cohen, L. et al. The visual word form area: Spatial and temporal characterization of an initial stage of reading in normal subjects and posterior split-brain patients. Brain, 123 (Pt 2), 2000, pp. 291-307.

5. Crow T. J., L. R. Crow, D. J. Done, and S. Leask. Relative hand skill predicts academic ability: global deficits at the point of hemispheric indecision. Neuropsychologia, 36 (12), 1998, pp. 1275-1281.

6. Dehaene, S. and L. Cohen. The unique role of the visual word form area in reading. Trends in Cognitive Sciences, 15 (6), 2011, pp. 254262.

7. Denny, K. and W. Zhang. In praise of ambidexterity: How a continuum of handedness predicts social adjustment. Laterality, 22 (2), 2017, pp. 181-194.

8. Dole, M., D. Méary, and O. Pascalis. Modifications of visual field asymmetries for face categorization in early deaf adults: A study with chimeric faces. Frontiers In Psychology, 20 (8), 2017, pp. 1-12, doi: 10.3389/fpsyg.2017.00030.

9. Dronkers, N. F. and R. T. Knight. Right-sided neglect in a left-hander: Evidence for reversed hemispheric specialization of attention capacity. Neuropsychologia, 27 (5), 1989, pp. 729-735, doi:10.1016/0028 3932(89)90118-8.

10. Ferneyhough, E.., D. Stanley, E. Phelps, and M. Carrasco. Cuing effects of faces are dependent on handedness and visual field. 
Psychonomic Bulletin \& Review, 17 (4), 2010, pp. 529-535, doi: 10.3758/PBR.17.4.529.

11. Franklin, R. and R. B. Adams. The two sides of beauty: Laterality and the duality of facial attractiveness. Brain and Cognition, 72 (2), 2010, pp. 300-305.

12. Grabowska, A. et al. Switching handedness: fMRI study of hand motor control in right-handers, left-handers and converted left-handers. Acta Neurobiologiae Experimentalis, 2012, 72 (4), pp. 439-451.

13. Hernandez, N. et al. (2009). Exploration of core features of a human face by healthy and autistic adults analyzed by visual scanning. Neuropsychologia, 47 (4), 2009, pp. 1004-1012.

14. Chen, W., C. H. Liu, and X. Fu. Asymmetry of left versus right lateral face in face re cognition. in Paiva, R., R. Prada, and W. Picard (Eds.): Affective Computing and Intelligent Interaction. ACII 2007. Lecture Notes in Computer Science, vol. 4738, Berlin, Heidelberg: Springer, 2007, pp. 712-713.

15. Jung, W. et al. Neural correlates of the eye dominance effect in human face perception: The left-visual-field superiority for faces revisited. Social Cognitive and Affective Neuroscience, 12 (8), 2017, pp. 1342 1350, doi: 10.1093/scan/nsx056.

16. Le Grand, R., C. J. Mondloch, D. Maurer, and H. P. Brent. Expert face processing requires visual input to the right hemisphere during infancy. Nature Neuroscience, 6 (10), 2003, pp. 1108-1112, doi:10.1038/nn1121.

17. Luh, K. E., J. Redl, and J. Levy. Left- and right-handers see people differently: Free-vision perceptual asymmetries for chimeric stimuli. Brain and Cognition, 25 (2), 1994, pp. 141-160. doi: 10.1006/brcg.1994.1028.

18. Maguinness, C and F. N. Newell. Recognising others: adaptive changes to person recognition throughout the lifespan. In Schwartz, B. L., M. L. Howe, M. P. Toglia and H. Otgaar (Eds.): What is Adaptive about Adaptive Memory? New York: Oxford University Press, 2014, pp. 231-257.

19. Nicholls, M. E. R., B. J. Wolfgang, D. Clode, and A. K. Lindell. The effect of left and right poses on the expression of facial emotion. Neuropsychologia, 40 (10), pp. 1662-1165, doi: 10.1016/S00283932(02)00024-6.

20. Oldfield, R. C. The assessment and analysis of handedness: the Edinburgh inventory. Neuropsychologia, 9 (1), 1971. pp. 97-113.

21. Perrett, D. I. In Your Face. The New Science of Human Attraction. New York, NY: Palgrave Macmillan 2010. 
22. Phillips, M. and A. David. Viewing strategies for simple and chimeric faces: an investigation of perceptual bias in normals and schizophrenic patients using visual scan paths. Brain and Cognition, 35, 1997, pp. 225-238.

23. Porac, C. and S. Coren. The dominant eye. Psychological Bulletin, 83 (5), 1976, pp. 880-97.

24. Reiss, M. and G. Reiss. Ocular dominance: Some family data. Laterality, 2 (1), 1977, pp. 7-16, doi: 10.1080/713754254.

25. Sheard, D. A. Test for Handedness. British Medical Journal, 6, 1(5022), 1957, p. 823.

26. Siebner, H. R. et al. Long-term consequences of switching handedness: a positron emission tomography study on handwriting in "converted" left-handers. The Journal of Neuroscience. 22 (7), 2002, pp. 28162825.

27. Thomas, C. et al. Reduced structural connectivity in ventral visual cortexin congenital prosopagnosia. Nature Neuroscience, 12 (1), 2008, pp. 29 - 31, doi:10.1038/nn.2224.

28. Yovel, G., A. Tambini, and T. Brandman. The asymmetry of the fusiform face area is a stable individual characteristic that underlies the left-visual-field superiority for faces. Neuropsychologia, 46 (13), 2008, pp. 3061-3068.

29. Zaidel, D. W. and J. A. Cohen. The face, beauty, and symmetry: perceiving asymmetry in beautiful faces. International Journal of Neuroscience, 115 (8), pp. 1165-1173.

30. Zaidel, D. W., A. C. Chen, and C. German. She is not a beauty even when she smiles: Possible evolutionary basis for a relationship between facial attractiveness and hemispheric specialization. Neuropsychologia, 33 (5), pp. 649-655. 